\title{
La imatge aristotèlica del paralític i la malaltia de la voluntat al poema 74 d'Ausiàs March*
}

\section{The Aristotelian paralysis metaphor and the weakness of the will in Ausias March's poem 74}

\author{
Salvador Cuenca i Almenar \\ salvador.cuenca@uv.es
}

Universitat Jaume I - Castelló

A Júlia Benavent

\begin{abstract}
Resum: El símil de la segona cobla de la cançó 74 d'Ausiàs March s'articula al voltant d'un mot agrest i poc poètic: «paralític». Dues obres de divulgació aristotèlica del dos-cents, la Summa Alexandrinorum i la Sententia libri Ethicorum de Tomàs d'Aquino, també empren «paralític» en el mateix context, a saber, per a referir-se a l'incontinent, és a dir, a qui pateix la malaltia de la voluntat. L'objectiu d'aquestes línies és mostrar la relació conceptual entre el poema $74 \mathrm{i}$ aquests dos textos basats en l'Ética nicomaquea d'Aristòtil.
\end{abstract}

Paraules clau: Ausiàs March, Aristòtil, Summa Alexandrinorum, Brunetto Latini, Tomàs d'Aquino

\begin{abstract}
The paralysis metaphor is crucial to the understanding of the second stanza of March's poem 74. Two 13th century works, essential for the dissemination of Aristotle's thought, namely, the Summa Alexandrinorum and Aquinas's Sententia libri Ethicorum, use the metaphor to adress the same moral issue: the akrasia or weakness of the will. This paper will examine the relationship between poem 74 and those two Aristotelian works.
\end{abstract}

Keywords: Ausias March, Aristotle, Summa Alexandrinorum, Brunetto Latini, Aquinas

\footnotetext{
* Aquest treball s'insereix dins del projecte PAPIIT de la Universidad Autónoma de México (Clave: IA400915) titulat «Recepción clásica y modernidad: autores de la Antigüedad clásica en la configuración del pensamiento ilustrado y romántico».
}

DATA PRESENTACIÓ: 17/05/2016 ACCEPTACIÓ: 25/05/2016 ·PUBLICACIÓ: 20/06/2016 
El símil de la segona cobla de la cançó 74 d'Ausiàs March s'articula al voltant d'un mot agrest i poc poètic: «paralític». Dues obres de divulgació aristotèlica del dos-cents, la Summa Alexandrinorum i la Sententia libri Ethicorum de Tomàs d'Aquino, l'empren també per a exemplificar el mateix problema moral: la malaltia de la voluntat. L'objectiu d'aquestes línies és mostrar la relació conceptual entre el poema 74 i aquests dos textos basats en l'Ética nicomaquea d'Aristòtil. Estretirem aquesta dependència en constatar l'aparició del terme «paralítics» al fragment corresponent (1102b) del Llibre del tresori de la traducció castellana de l'Ėtica nicomaquea inventariada per Schiff a la Bibliothèque du Marquis de Santillane (Schiff 1905: 31-4).

Diversos erudits han remarcat que March es val de conceptes tomistes (Pagès 1912, 1925), augustinians (Mestre 2009, 2014), aristotèlics (Archer 1991a/1991b, Cabré 1996) i senequians (Martínez 1998) per a descriure els problemes morals relacionats amb la praxi de l'amor humà. La qüestió tractada pel poeta valencià al poema 74 és el problema de la malaltia o feblesa de la

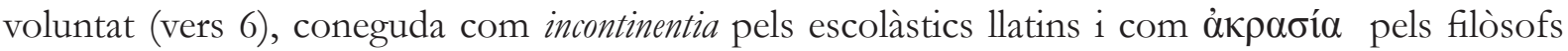
grecs. En el context del poema 74: el jo líric sap què ha de fer, però no ho pot fer, perquè pateix la

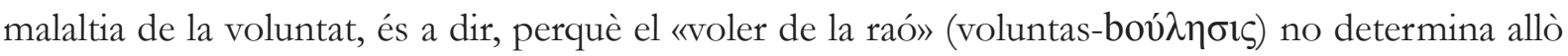
que s'ha de fer i «és vençut» (vers 14) per les parts irracionals de l'apetit (vers 24). La victòria de

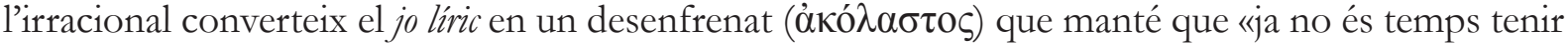
frens al voler» (vers 5) i que «malament viu qui en mal fer no té frens» (vers 32). El desenfrenament contumaç acaba per transmutar l'incontinent en un intemperat, amb la consegüent reducció del jo líric a ximple titella material moguda des dels astres pels fats, motors de les etèries esferes planetàries, als quals s'encomana des del primer vers. Així com els fats causen el moviment dels cels, així també provoquen els actes del cos del jo líric, que ha perdut la capacitat d'elegir racionalment (electio-

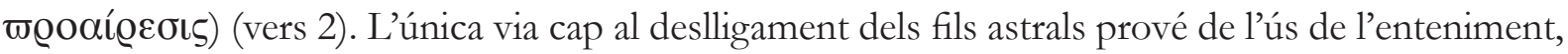
que «ha gran debat ab lo voler del cos» (concupiscentia-غ̇ं $\imath \theta v \mu i ́ \alpha)$ (vers 23). El jo líric, nogensmenys, no pot determinar el resultat del debat i, «penjant lo temps» (vers 24), es deixa dur per l'apetit i pels influxos astrals que exciten la matèria corporal.

El canemàs conceptual sobre el qual brodem aquesta interpretació està desenrotllat a l'últim capítol del primer llibre de l'Ética nicomaquea d'Aristòtil. Llegim les dues primeres cobles de la cançó marquiana, segons l'edició a cura de Pere Bohigas, revisada per Soberanas i Espinàs, ressaltant els conceptes ètics fonamentals:

\footnotetext{
Als fats coman tot quant serà de mi, puys só estolt de ma elecció; mon seny és mort, a qui Déu no perdó, puys al començ de tot me derrenclí. Ja no és temps tenir frens al voler, malalta és ma bona voluntat,
} 
e vaig en loch on no vull ser portat: só descontent de tot quant pusqua fer.

Sí com al hom no li basta poder, paralitich, quant és en peus levat, anar al loch on vol ésser anat, ans cau, a tort va contra son mester; ne pren a mi que faç lo que no $\mathrm{m}$ plau y aquell voler de la rahó 's vençut: e si ll complach mon delit és perdut, per què sens cor faç quant de mi vejau.

March podia haver obtingut els coneixements d'Aristòtil de resums com la Summa Alexandrinorum, traduïda als Livres dou Tresor per Brunetto Latini, i que podia haver-se trobat a la la biblioteca del pare d'Ausiàs (Archer 1991a: 9). Podem, per tant, recórrer al text de la Summa i del Tresor per a recercar la font d'inspiració marquiana. Contrastem en primer lloc el capítol quart segon llibre del Tresor (Latini 2007: 342-344), segons l'edició curada per Beltrami:

\footnotetext{
Et tu dois savoir que en l'arme sont aucune foiz contraires movemenz, ausi come ou cors quant l'un membre se muet et est paralitiques, qu'il convient mouvoir contre nature; mais ceste contrarieté est manifeste ou cors et privee en l'arme.
}

Pel que fa a la font llatina de la translació francesa, Sonia Gentili (2014: 4) observa que l'exemple del paralític no està present en la primera de les tradicions textuals de la Summa, aquella triada com a base de l'edició de Marchesi, i sí en la segona. En la primera Gentili percep un salt per homoioteleuton:

Verumtamen fortassis oportet non ignorare in anima aliquid esse coniurans et contradicens rationi, quemadmodum in membris hoc quidem manifestum contra in anima et occultatur.

En canvi, l'equiparació amb el paralític sí que es troba en la segona de les famílies de la Summa. Marquem entre claudàtors el text omés pel salt d'igual a igual en la primera tradició textual:

\footnotetext{
Oportet enim non ignorare in anima aliquid esse contrariis et contradictionis rationi quemadmodum [in membris paraliticis videmus esse causam motivam in partes oppositas motui nature. Verum] in membris hoc quidem manifestum, in anima vero occultum.
}

Pel que fa a aquest fragment concret, podem acostar el text català traduït per Guillem de Copons, al dependre de la versió francesa, segurament la del manuscrit R de Carmody (Latini 1971: 30), a la segona tradició textual de la Summa, perquè es conserva l'analogia amb el paralític (Latini 1976: 116-7): 
En l'ànima són algunes vegades contraris moviments, axí com és en lo cors, com lo I membre se mou e és paralítich, lo qual cové moure contra natura; mas aquesta contrarietat és manifesta al cors e privada a l'ànima.

Cal recordar que l'editor Wittlin (1976: 117) hi apunta que el manuscrit 232 de la Biblioteca Ep iscopal de Vic trasllada millor la comparació anterior: «axí com lo cors, quant la un membre, estant paralítich, se mou contra natura». Podem corroborar l'observació de Wittlin amb la lectura atenta del foli 7 recto del manuscrit vigatà:

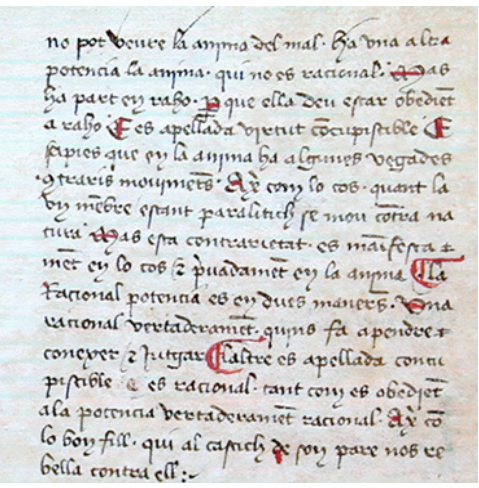

D’altra part, Amadeu Pagès insistia en la importància de rastrejar les petges tomistes en la lírica marquiana (Pagés 1912). Si seguim aquesta indicació, hem d'adreçar-nos a la Sententia libri ethicorum, en la qual l'Aquinat comenta l'Ética nicomaquea d'Aristòtil. Cal apuntar que la utilització literària de la Sententia tomasiana no seria una raresa d'Ausiàs, ja que Giovanni Boccaccio també l'emprà per a bastir les reflexions morals dels seus personatges, com ara en Decameron II 8 i X 7 (Ellero 2015). A més, es conserva un manuscrit autògraf del Boccaccio amb les glosses de l'Aquinat copiades al marge, a saber, el ms. A 204 Inf. de la Biblioteca Ambrosiana de Milà (Cesari 1968, Barsella 2012).

En glossar el pas 1102b, segons la paginació canònica de Bekker, l'Aquinat ens ofereix una altra deu possible del símil marquià, a saber, la Sententia, liber I, lectio 20, n. 8 (1969: 72):

\begin{abstract}
Et ideo subdit exemplum, quod sicut quando corporis membra sunt dissoluta, quia scilicet non possunt omnino contineri a virtute corporis regitiva, sicut accidit in paraliticis et ebriis, qui moventur in partem sinistram, quando homines eligunt moveri in dextram; ita etiam firmiter verum est ex parte animae in incontinentibus quod moventur ad contraria eorum quae ratio eligit. Sed hoc non ita apparet in partibus animae, sicut in partibus corporis. Quia in partibus corporis manifeste videmus quando aliquid inordinate movetur, sed in partibus animae non ita manifeste hoc videmus.
\end{abstract}

Cal remarcar que a la Translatio lincolniensis no apareix l'equivalent al terme «paralític», fidel al text grec de l'Ética nicomaquea (1102b 14-20): 
Salvador Cuenca Almenar. La imatge aristotèlica del paralític i la malaltia de la voluntat al poema 74

d'Ausiàs March

\begin{abstract}
Incontinentis enim et continentis, racionem habens laudamus. Recte enim et ad optima, deprecatur ratio. Videtur in ipsis et aliud quid preter racionem innatum quod adversatur et obviat racioni. Firmiter enim quemadmodum dissolute corporis particule in dexteram preeligencium movere e contrario in sinistram prave feruntur, et in anima sic; ad contraria enim, motus incontinencium (1972: 161).

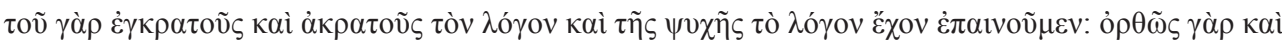

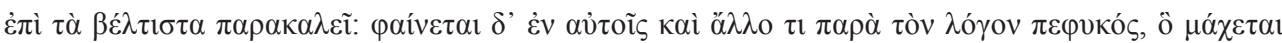

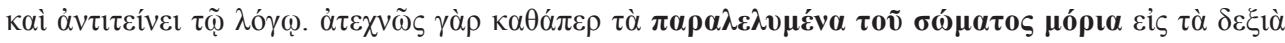

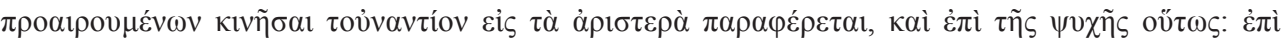

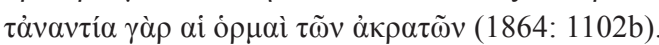

En canvi, a diferència de l'Aristoteles latinus, l'arromançament que, segons Schiff, hauria pertangut a la biblioteca del Marqués de Santillana, sí que reporta el terme «paralític» en el fragment ací analitzat (BNE MSS/ 10268: 18r):

E paresçe del continente e non continente loaremos aquella parte que ha entendimiento; que'l entendimiento aderesçará a las cosas escogidas. E paresçe en ellos otra cosa natural afuera del entendimiento que ${ }^{1}$ contraria e resiste al entendimiento con fortaleza. E es ansí como los paralíticos del un lado del cuerpo, ca, si quieren moverse a la parte derecha, trastórnanse a la parte siniestra malamente. Ansí en la ánima, el movimiento de los yncontinentes es al contrario.

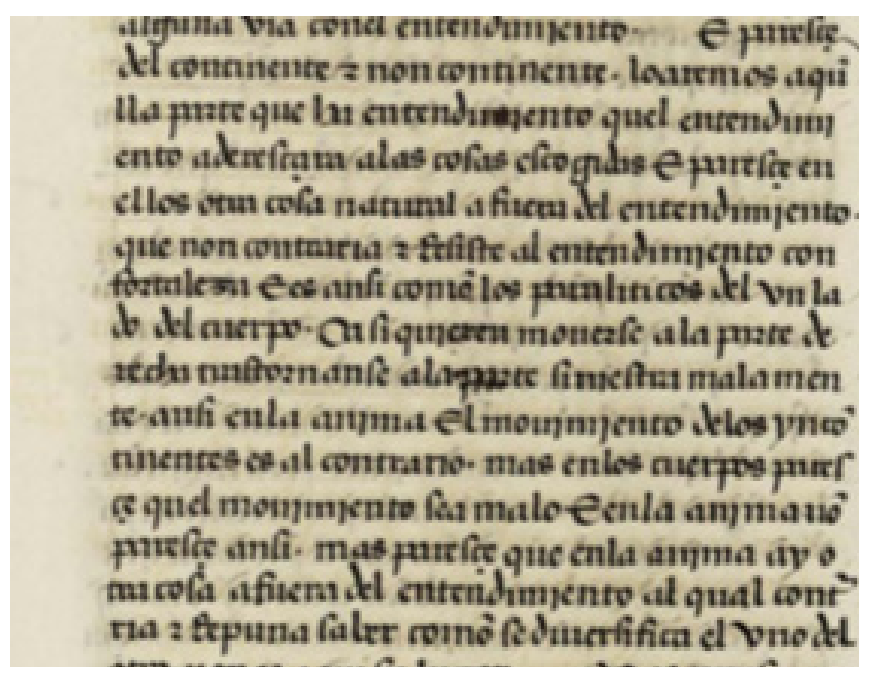

Cal afegir que la traducció del Príncep de Viana no esmenta l'adjectiu «paralíticas» i sí «resolvidas» per a complementar el sintagma «partes del cuerpo» com a correlat de l'incontinent que es deixa caure en la inèrcia del desig (BNE MSS/ 6984 f. 46r-v):

Parece ser en ellos una otra cosa sin razón, que contraria e resiste a la razón. Ca ansí como las partes resolvidas del cuerpo, si a la diestra parte moverlas quieres, a la diestra van, assí en el ánimo en contrario se mueven los appetitos de los incontinentes.

1 Cancel lem «non» per a preservar el sentit de l’argumentació aristotèlica.

SCRIPTA, Revista internacional de literatura i cultura medieval $i$ moderna, núm. 7 / juny 2016 / pp. 42 - 51 
Tampoc no s'empra el mot «paralític» sinó «coixo» al passatge corresponent del Compendi de l'Ètica nicomaquea, que circulava per la península ibèrica en aragonés, castellà i català a la segona meitat del segle XV (Cuenca 2012: 49):

\begin{abstract}
Quant al que cové saber al moral és [que] de aquestes dos parts de rahó, la una és racional per essència, l'altra és racional per participació. E la que és racional per essència persuadeix e convida a la part inferior al bé e tota la differència dels hòmens ser virtuosos o viciosos stà en la differència o concòrdia de estes dos. (...) El incontinent té lessa la part inferior e és axí com lo coixo que eleix de anar dret e per la lesió de aquella part és portat en lo sinistre. E no és menor la malaltia de la ànima que la del cors, possat no sia visible.
\end{abstract}

Per a arredonir aquest panorama general dels usos del terme «paralític», cal indicar breument les diferències entre la utilització que se'n fa al poema 74 i al poema 101, les dues úniques aparicions del mot en qüestió a la lírica ausiasmarquiana. Deixarem a banda el problema de l'atribució del poema 101 al vat valencià, sospitosa per a Ferraté (1979: 25) i acceptable per a Archer (1993). Recordem, no obstant, que l'erudit londinenc (1993) afirma que «difícilmente se encuentran motivos objetivos para rechazar el poema». D'altra part, la tradició lírica catalana del cinc-cents confiava en l'autenticitat de la cançó 101, com palesa el «Cant d'amors» de Pere Serafí (Vallsalobre 1999: 229)

Comparem, doncs, ambdues composicions. Al poema 74, amb la imatge del paralític s'exemplifica la malaltia de la voluntat, és a dir, la incontinència que impedeix romandre en l'elecció justa

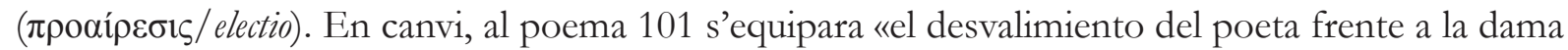
y la angustia del paralítico enfermo en tierra extraña, incapaz de indicar con gestos el mal que lo desazona» (Rico 1982: XXII). Cal recordar que, segons Rico, la font de la imatge del paralític brolla del sermó «que dio maestre Pedro Marín al Conde (de Haro)». Remembrem l'entrada triomfal del 101:

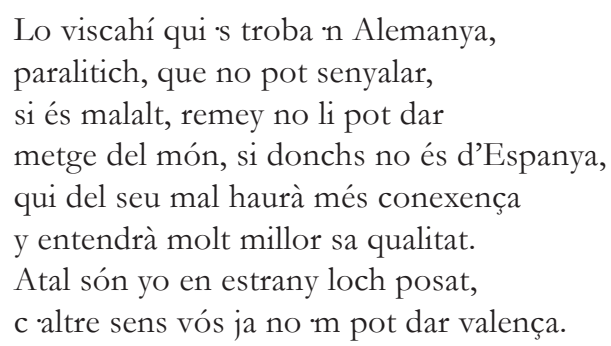

La diferència amb el poema 74 quant al referent del terme analogat és cridanera: el «paralític» del poema 101 no és un incontinent, sinó un impotent. No es refereix al malalt dominat pel desig irracional, sinó a qui és incapaç d'indicar quina és la seua dolença a qui no entén els seus signes lingüístics. I el jo líric solament pot ser comprés per la «bell’ab bon seny», sols ella li pot dar valença. 
No obstant la divergència en l'ús, es poden advertir dues semblances entre ambdós poemes, una de forma i l'altra de fons. Quant a la formal, es percep la col locació ajustada del mot quatrisíl lab en el primer hemistiqui del segon vers de les cobles en què apareix. Pel que fa al contingut, en ambdues composicions l'apetit irracional triomfa sobre l'enteniment a l'hora de determinar la voluntat; semblança quasi anecdòtica, ja que es tracta d'un dels motius més repetits a la lírica ausiasmarquiana.

La tornada del poema 74 ens permet aproximar-nos a una primera interpretació del seu tractament de la incontinència i resemantitzar-ne les dues primeres cobles de la manera que exposarem a continuació. La feblesa de la voluntat es manifesta quan el jo líric imagina què podria fer el referent del senyal. Si no hi fantasieja, la malaltia es manté en un estat latent. Si ho fa, la dolença es manifesta. Si se succeeixen les imatges del llir entre cards, el seu curs indeturable anorrea la voluntat del jo líric, que perd el control («poder» versos 9 i 51) sobre els seus actes, en tant que la voluntat o «voler de

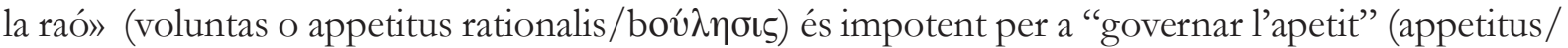
ọ̋ $\xi ı$ เ ) (vers 52). Podem inferir aquesta interpretació a partir dels dos últims versos de la poesia d'acord amb el manuscrit K (MSS/2025 Biblioteca de Catalunya): «tot act'és prop de lla on és poder,/ si el voler governa l'apetit». A banda, a nivell d'inspiració conceptual, podem tornar a la Sententia libri ethicorum (lib. 31.4 n. 2) de Tomàs per a entendre que la voluntat és una de les espècies de l'apetit, a saber, l'apetit racional (Tomás 1969: 129):

\footnotetext{
non omne illud cuius principium est intra, etiam cum scientia circumstantiarum, est voluntarium; potest enim contingere quod illud principium quod est intra non sit appetitus rationalis qui dicitur voluntas a qua denominatur voluntarium, sed aliqua passio appetitus sensitivi, puta ira vel concupiscentia vel aliquid aliud huiusmodi
}

Avui sabem que la llum dels estels zodiacals ve d'altres segles i que es percep molt després, potser com un text del passat. Ve d'altres segles segons fat, ventura o predestinació i encadena els fets i les imatges a l'avenir. Al poema 74, els fets del jo líric es desvien de la «bona fi» i precipiten el present envers la tristor del «dan avenidon» (versos 41-43). El fat encadena a la matèria $\mathrm{i}$ al deixar-se dur de l'apetit sensitiu; és més, el jo líric demana encadenar-s'hi. En una descripció retòrica de la inèrcia animal, el jo líric elegeix no elegir: «als fats coman tot quant serà de mi». El gran delit se sent abans d'encomanar la cadena i encadenar-se. Després, les baules de les imatges vertides afeixuguen el jo líric, esclau del desig i de la llum que prové d'altres temps. La manifestació del desig imaginat cancel•la el delit anterior i encadena els moviments del cos als dels astres. Cadenes en forma de llum que es propaga a través de l'èter. Estreles vistes segles després d’haver emés la llum. Inèrcies dels cossos sens èter. «Penjant lo temps», apetits sens raons. 
Salvador Cuenca Almenar. La imatge aristotèlica del paralític i la malaltia de la voluntat al poema 74 d'Ausiàs March

\section{Bibliografia}

Archer, Robert (1991a), «Ausiàs March i els tòpics», Anuari de l'Agrupació Borrianenca de Cultura, II, pp. 5-17.

. (1991b), «Una font aristotèlica d'Ausiàs March», en Ferrando, Antoni; Hauf, Albert G. (eds.), Miscel lània Joan Fuster. Estudis de Llengua $i$ Literatura, IV, Valencia/Barcelona, Universitat de València / Publicacions de l'Abadia de Montserrat, pp. 59-74.

. (1993), «Ausiàs March en sus manuscritos: reevaluación de tres problemas fundamentales», Hispanic, pp. 43-57 (on-line en Biblioteca Virtual Joan Lluís Vives, http:/ / www.cervantesvirtual. com/nd/ark:/59851/bmc320d9 [17 de maig de 2016])

- (1997), Ausiàs March. Obra completa, 2 vols., Barcelona, Barcanova.

Aristòtil (1972), Ethica nicomachea. Translatio Roberti Grosseteste Lincolniensis. Recensio pura, a cura de Gauthier, R. A., Brussel les / Brill, Leiden / Desclée de Brouwer

—. (1894), Ethica Nicomachea, ed. J. Bywater, Oxford, Clarendon Press, (on-line en http://data. perseus.org/citations/urn:cts:greekLit:tlg0086.tlg010.perseus-grc1:1102b [17 de maig de 2016])

Barsella, S (2012), «I “marginalia”di Boccaccio all'EticaNicomachea di Aristotele (Milano, Biblioteca Ambrosiana A 204 Inf.)», en Boccaccio in Americam a cura de Filosa, E. \& Papio, M., Ravenna, Longo, pp. 143-155

Cabré, Lluís (1996), «Aristotle for the layman: sense perception in the poetry of Ausias March», Journal of the Warburg and Courtauld Institutes, 59, pp. 48-60.

- (1998), «Notas sobre la memoria de Santillana y los poetas de la Corona de Aragón», en Deyermond, Alan (ed.), Cancionero. Studies in Honour of Ian Macpherson, Londres, Queen Mary and Westfield College, pp. 25-38.

Cabré, Lluís; Ferrer, Montserrat (eds.) (2012), Cens de traduccions al català medieval fins a 1500, en http://translatdb.narpan.net/ [9 de juny de 2012].

Cantavella, Rosanna (2010), «Sobre la poesía antifemenina de Ausiàs March: el poema 71», Revista de Literatura Medieval, 22, pp. 85-104.

Cesari, A. M. (1968) «L'Etica di Aristotele del codice Ambrosiano A204 inf.: un autografo del Boccaccio», Archivio Storico Lombardo, XCIII-XCIV, pp. 69-100.

Colomer i Pous, Eusebi (1997), El pensament als Països Catalans durant l'Edat Mitjana i el Renaixement, Barcelona, Institut de Estudis Catalans / Abadia de Montserrat.

Cuenca i Almenar, Salvador (2005), «El seny contra l'enteniment al poema 4 d'Ausiàs March», Llengua \& Literatura, 16, pp. 33-55.

—. (2008), «La fantasia i el poema 18 d'Ausiàs March», Llengua \& Literatura, 19, pp. 27-47.

—. (2012), «L'Ètica nicomaquea d'Aristòtil en un compendi català del segle XV», Anuari de la

SCRIPTA, Revista internacional de literatura i cultura medieval i moderna, núm. 7 / juny 2016 / pp. 42 - 51 
Salvador Cuenca Almenar. La imatge aristotèlica del paralític i la malaltia de la voluntat al poema 74 d'Ausiàs March

Societat Catalana de Filosofia, 23, pp. 7-119

Cursi, M. (2013) «Giovanni Boccaccio- Autografi», en Autografi dei letterati italiani. Leorigini e il Trecento, a cura de G. Brunetti, M. Fiorilla, M. Petoletti, Roma, Salerno editrice, pp.47-53.

Di Girolamo, Costanzo (ed.) (2004), Ausiàs March. Páginas del Cancionero, València, Pre-textos.

Escobar, Ángel (2015), «Hacia una caracterización de la transmisión aristotélica en la Cataluña medieval», Anuario de Estudios Medievales, 45/1, pp. 299-330

Ellero, Maria Pia (2015), «Lisa e l'aegritudo amoris. Desiderio, virtù e fortuna in Decameron, II 8 E X 7», en Boccaccio 1313-2013, a cura de Francesco Ciabattoni, Elsa Filosa, Kristina Olson, Ravenna, A. Longo Editore.

Ferraté, Joan (1979), «Pròleg» en March, Ausiàs, Poesia, Barcelona, Edicions 62.

Gentili, Sonia (2014), «L'edizione dell'Etica in volgare attribuita a Taddeo Alderotti: risultati e problemi aperti», en Aristotele fatto volgare. Tradizione aristotelica e cultura volgare nel Rinascimento, a cura de David A. Lines \& Eugenio Refini, Pisa, Edizioni ETS.

Icardo, Juan Antonio (2010), «La presencia de Aristóteles en Ausiàs March», en Bellveser, Ricard (ed.), La poesia d'Ausiàs March i el seu temps, València, Institució Alfons el Magnànim, pp. 373-378.

Latini, Brunetto (1971-1976), Llibre del tresor, ed. a cura de Wittlin, K., Barcelona, Barcino, 2 vols.

—. (2007), Tresor, ed. a cura de Beltrami, E., Torí, Einaudi.

Lloret, Albert (2013), Printing Ausiàs March: material culture and Renaissance poetics, Madrid, Centro para la Edición de los Clásicos Españoles.

Lohr, Charles H., (1967), «Medieval Latin Aristotle Commentaries», Traditio, 23, pp. 313-413.

. (1995), Latin Aristotle commentaries, III: Index initiorum - Index finium, Florencia, Leo S. Olschki Editore.

March, Ausiàs, (2000) Poesies, a cura de Pere Bohigas, revisada per Amadeu J. Soberanas i Noemí Espinàs. Pròleg de Lola Badia. Bibliografia elaborada per Joan Santanach i Vicent Martines. Barcelona, Barcino.

Martínez Romero, Tomàs, (1998) «Un clàssic entre els clàssics. Sobre traduccions i recepcions de Sèneca a l'època medieval», València / Barcelona, Institut Interuniversitari de Filologia Valenciana / Publicacions de l'Abadia de Montserrat.

—. (2009), «Bruni, Martí de Viciana i el pròleg a la traducció de l'Econòmica aristotèlica», Caplletra, 47, pp. 41-54.

Mestre, Marina (2009), «El papel de la antropología augustiniana en la poesía de Ausias March», Criticón, 107, pp. 11-27.

—_. (2014), Ausiàs March. L'impossible orthodoxie de l'être, Madrid, Casa de Velázquez.

Minio-Paluello, Lorenzo (1972), Opuscula. The Latin Aristotle, Amsterdam, Adolf M. Hakkert.

SCRIPTA, Revista internacional de literatura i cultura medieval i moderna, núm. 7 / juny 2016 / pp. 42 - 51 
Salvador Cuenca Almenar. La imatge aristotèlica del paralític i la malaltia de la voluntat al poema 74 d'Ausiàs March

Pagès, Amédée (1912), Auzias March et ses prédécesseurs: Essai sur la poésie amoureuse et philosophique en Catalogne aux XIVe et XVe siècles, Paris, Librairie Ancienne Honoré Champion.

—. (1925), Commentaire des poésies d'Auzias March, París, Librairie Ancienne Honoré Champion.

Ramírez i Molas, Pere (1980), «El problemàtic cant 128 d'Ausiàs March i la tradició manuscrita», Estudis Universitaris Catalans, 24, Barcelona, Curial, pp. 497-512.

Rico, Francisco (1982), Primera cuarentena y Tratado general de literatura, Barcelona, El Festín de Esopo.

Robles Carcedo, Laureano (1979), «Aristoteles Latinus. Repertorio de manuscritos españoles», en Actas del V Congreso internacional de filosofía medieval, vol. I, Madrid, Editora Nacional, pp. 333-460.

—_. (1992), Tomás de Aquino, Salamanca, Universidad de Salamanca.

Rubio, Josep E. (2009), «Salvar Aristòtil? La teologia davant la cultura pagana a la tardor medieval», eHumanista, 13, pp. 173-194.

Schiff, Mario (1905), La Bibliothèque du Marquis de Santillane, París, Emile Bouillon.

Tomàs d'Aquino (1969), Sententia libri Ethicorum, ed. a cura de l'Ordre dels Predicadors, Roma, Ad Sanctae Sabinae.

Vallsalobre, Pep (1999), «Lectura de 'Cant d'Amors' de Pere Serafí: una art poética del renaixement hispànic», dins Rafanell, A. \& P. Balsalobre, Estudis de Filologia Catalana, Barcelona, Publicacions de l'Abadia de Montserrat (on-line en http://www3.udg.edu/vell/ilcc/Eiximenis/html_ eiximenis/pdf\%20eiximenis/Cant\%20d'amors.pdf ([consultat 119 de maig de 2016]) 